\title{
Liver kinase B1 restoration promotes exosome secretion and motility of lung cancer cells
}

\author{
CHENG ZHANG $^{1,3^{*}}$, XIANG XIAO $^{2 *}$, MINYI CHEN $^{1}$, HITHAM ALDHAREE $^{1}$, \\ YANFANG CHEN $^{2}$ and WEIWEN LONG ${ }^{1}$
}

\begin{abstract}
Departments of ${ }^{1}$ Biochemistry and Molecular Biology, and ${ }^{2}$ Pharmacology and Toxicology, Wright State University, Dayton, OH 45435, USA; ${ }^{3}$ Department of Spine Surgery, The First Affiliated Hospital, University of South China, Hengyang, Hunan 421001, P.R. China
\end{abstract}

Received August 10, 2017; Accepted October 24, 2017

DOI: $10.3892 /$ or.2017.6085

\begin{abstract}
Liver kinase B1 (LKB1) regulates a variety of cellular functions, including cell polarity, energy metabolism and cell growth, by targeting multiple signaling pathways such as AMPK/mTOR and p53. LKB1 functions as a tumor suppressor in sporadic cancers including lung cancer. Extracellular vesicles such as exosomes secreted by cancer cells modulate the tumor microenvironment and progression by targeting both tumor cells (autocrine actions) and other types of cells associated with tumors (paracrine actions). While the roles of LKB1 in cellular signaling in general is well-studied, its specific role in exosome-mediated signaling remains to be explored. To this purpose, we reintroduced LKB1 into H460 and A549 lung cancer cells that are endogenously deficient in LKB1 expression. Notably, we found that while restoration of LKB1 significantly reduced lung cancer cell growth as expected, it greatly promoted cell motility and enhanced the release of exosomes. In addition, exosomes isolated from H460 cells with stable restoration of LKB1 had much higher ability in stimulating lung cancer cell migration than did those from $\mathrm{H} 460$ cells lacking LKB1. Mechanistically, restoration of LKB1 in H460 cells inhibited cellular expression and exosomal secretion of migration-suppressing microRNAs (miRNAs), including miR-125a, miR-126 and let7b. Taken together, the present study revealed a new role for LKB1 in promoting cell motility by downregulating migration-suppressing miRNA expression and exosome secretion.
\end{abstract}

Correspondence to: Dr Weiwen Long, Department of Biochemistry and Molecular Biology, Wright State University, 112 Diggs Laboratory, 3640 Colonel Glenn Hwy., Dayton, OH 45435, USA

E-mail: weiwen.long@wright.edu

${ }^{*}$ Contributed equally

Key words: LKB1, cell migration, exosome secretion, migrationsuppressing miRNAs, lung cancer

\section{Introduction}

Liver kinase B1 (LKB1), also known as serine/threonine kinase 11 (STK11), plays critical roles in cell growth, differentiation, polarity and migration $(1,2)$. LKB1 signaling controls energy metabolism and tissue homeostasis, and deletion of the LKB1 gene is embryonic-lethal (3). LKB1 signaling is also highly involved in human diseases. Germ-line mutations in LKB1 are associated with the predisposition of Peutz-Jeghers syndrome (4). Loss of LKB1 expression by either somatic mutations or promoter hypermethylation is frequently identified in sporadic cancers including lung cancer (1). Disruption of LKB1 gene function promotes tumor progression in multiple animal tumor models (1). As such, LKB1 is considered as a tumor suppressor in general. Mechanistically, LKB1 regulates cellular events by targeting multiple critical signaling pathways, including AMPK/mTOR, p53 and PTEN/Akt (5).

Accumulating evidence has demonstrated that extracellular vesicles, such as exosomes and microvesicles, carry and transmit cellular molecules and signals, and mediate cell-cell communications (6). In cancers, this process is shown to be important for modulating the tumor microenvironment, in which tumor cells and tumor-associated cells intercommunicate to control tumor progression (7). Exosomes secreted by cancer cells can target both tumor cells (autocrine actions) and other types of cells associated with tumors (paracrine actions). Of the molecules contained in exosomes, microRNAs (miRNAs) have received the most attention due to their diverse and critical roles in tumor progression and their highly potential diagnostic and therapeutic applications in cancer treatment (8). Notably, while intracellular LKB1 signaling has been well-studied, its roles in extracellular vesicle-mediated cell signaling remain unclear. In the present study, we found that restoration of LKB1 in LKB1-deficient H460 and A549 lung cancer cells markedly enhanced motility and increased secretion of exosomes. Importantly, in comparison with those from $\mathrm{H} 460$ cells with LKB1 deficiency, exosomes secreted by $\mathrm{H} 460$ cells with restoration of LKB1 had highly increased ability to promote cancer cell migration. Mechanistically, restoration of LKB1 in $\mathrm{H} 460$ cells inhibited cellular expression and exosomal secretion of migration-suppressing miRNAs, including miR-125a, miR-126 and let7b. 


\section{Materials and methods}

Generation of a construct for lentiviral expression of human LKB1 ( $p C D H$-LKB1). The pCDNA3-Flag-LKB1 construct was a gift from Dr Lewis Cantley (Addgene, plasmid \#8590; Cambridge, MA, USA). pCDH-LKB1 was generated by inserting the Flag-LKB1 fragment released from pCDNA3-Flag-LKB1 into a lentiviral expression vector pCDH-CMV-MCS-EF1-Puro (System Biosciences, Mountain View, CA, USA) by EcoRI digestion. The resulting clone was verified by DNA sequencing.

Cell culture. Cell lines 293T, H460 and A549 were purchased from the American Type Culture Collection (ATCC; Manassas, VA, USA). 293T cells were cultured in Dulbecco's modified Eagle's medium supplemented with $10 \%$ fetal bovine serum (FBS). H460 and A549 cell lines were maintained in RPMI1640 medium supplemented with $10 \%$ FBS. All the culture media and supplements were purchased from Invitrogen (Carlsbad, CA, USA).

Generation of $H 460$ and A549 cell pools stably expressing LKB1 by lentiviral transduction. Production of pseudolentiviral particles and stable cell pools by lentiviral transduction was performed by following the manufacturer's instructions (System Biosciences). Pseudolentiviruses were produced in 293T cells by co-transfecting pCDH-LKB1 (or pCDH-CMVMCS-EF1-Puro control vector) and pPACK packaging plasmid mix (System Biosciences) using FuGENE HD reagent (Roche Applied Biosciences, San Diego, CA, USA). Pseudoviral particles were harvested $48 \mathrm{~h}$ post-transfection and concentrated using PEG-it ${ }^{\mathrm{TM}}$ Virus Precipitation Solution following the manufacturer's instructions (System Biosciences). H460 or A549 lung cancer cells were transduced with the prepared lentiviruses in the presence of Polybrene $(5 \mu \mathrm{g} / \mathrm{ml})$ in culture media. Two days post-transduction, the cells were split and selected by puromycin $(1 \mu \mathrm{g} / \mathrm{ml})$ for 10 days for obtaining stable cell pools.

Western blotting. Cells were lysed with EBC lysis buffer [50 mM Tris, pH 7.5, $150 \mathrm{mM} \mathrm{NaCl,} \mathrm{0.5 \%} \mathrm{NP-40,} 1 \mathrm{mM}$ phenylmethylsulfonyl fluoride (PMSF), $1 \mathrm{mM}$ complete protease inhibitors (Roche Diagnostics, Mannheim, Germany), $10 \mathrm{mM}$ NaF, $1 \mathrm{mM}$ sodium orthovanadate]. Total cell lysates were cleared by centrifugation at 13,000 rpm for $10 \mathrm{~min}$ at $4^{\circ} \mathrm{C}$. The supernatant (protein lysate) was added with $5 \mathrm{X}$ Laemmli sample buffer and boiled at $95^{\circ} \mathrm{C}$ for $5 \mathrm{~min}$ for denaturing the proteins. Protein samples were resolved on $10 \%$ sodium dodecyl sulfate-polyacrylamide gel electrophoresis (SDS-PAGE) gels and were transferred to nitrocellulose membranes. Western blotting was performed by first blocking nitrocellulose membranes with 5\% non-fat milk in PBS-T buffer for $30 \mathrm{~min}$, followed by overnight incubation with the primary antibody at $4^{\circ} \mathrm{C}$ and $1 \mathrm{~h}$ incubation with appropriate secondary antibody at room temperature. The western blotting was visualized by enhanced chemiluminescent (ECL) reagent (Pierce, Rockford, IL, USA). Primary antibodies used in western blotting were: anti-LKB1 (Cell Signaling Technology, Danvers, MA, USA) and anti-actin (Sigma-Aldrich, St. Louis, MO, USA).
Cell morphology analysis. H460 and A549 stable cell pools were plated into a 6 -well plate at a density of $1 \times 10^{5}$ cells/well. When cell density reached $\sim 80 \%$ confluence, cells were photographed using a phase-contrast microscope/camera system (Carl Zeiss, Jena, Germany) at a magnification of x40.

Cell migration assay. Cell migration was analyzed using a modified two-chamber Transwell system (BD Biosciences, San Jose, CA, USA) following the procedures previously described (9). Briefly, cells were detached by trypsin-EDTA, washed once with $1 \mathrm{X}$ phosphate-buffered saline (PBS), and then resuspended in serum-free medium. Complete culture media $(0.5 \mathrm{ml})$ was added to each bottom well. Cells $\left(1 \times 10^{5}\right)$ were added to each Transwell insert and allowed to migrate for $16 \mathrm{~h}$ in a $37^{\circ} \mathrm{C}$ cell incubator. Cells in the upper surface of the Transwell were removed using cotton swabs. Migrated cells that had attached to the undersurface were fixed with $4 \%$ paraformaldehyde for $10 \mathrm{~min}$ and stained with crystal violet solution ( $0.5 \%$ in water) for $10 \mathrm{~min}$. Cells were counted under microscopy at a magnification of $\times 100$.

Cell colony formation and MTS cell proliferation assays. Cell colony formation assay was performed using Millipore transformation assay kit (Millipore, Bedford, MA, USA) following the manufacturer's protocol. Briefly, a base agar layer containing $0.8 \%$ agarose was first prepared in a 24 -well plate. Cell solution $(0.25 \mathrm{ml})$ resuspended in complete culture media containing $0.4 \%$ agarose was then added to the top of the base agar layer (1,200 cells/well). Cells were incubated for 2-3 weeks at $37^{\circ} \mathrm{C}$ with $5 \% \mathrm{CO}_{2}$ and with medium exchange every 3 days. Colonies were photographed using a Primovert microscope (Carl Zeiss, Jena, Germany) equipped with a Motic camera/software system. Colonies were then quantified either by staining with $0.5 \mathrm{ml}$ of cell stain solution overnight and counting, or by adding cell quantification solution $(0.08 \mathrm{ml} /$ well $)$ and measuring absorbance at $490 \mathrm{~nm}$ using Synergy H1 microplate reader (BioTek Instruments, Inc., Winooski, VT, USA).

Cell proliferation was determined using the CellTiter 96 AQueous One Solution Cell Proliferation Assay kit (Promega, Madison, WI, USA), following the manufacturer's instructions.

Exosome release and isolation. For induction of exosome production and release, $\mathrm{H} 460$ cells were cultured in complete media until 80-90\% confluence, and then washed 3 times with PBS and cultured for $24 \mathrm{~h}$ in serum-free media. The conditioned media were collected and centrifuged at $300 \mathrm{xg}$ for $10 \mathrm{~min}$ to remove dead cells. The supernatant was centrifuged at $2,000 \mathrm{x} \mathrm{g}$ for $20 \mathrm{~min}$ to remove cell debris, followed by centrifugation at $20,000 \mathrm{x} \mathrm{g}$ for $30 \mathrm{~min}$. The supernatant was then collected and exosomes were pelleted by centrifugation at $169,000 \mathrm{x} g$ for $90 \mathrm{~min}$. The exosome pellet was resuspended in 1X PBS and analyzed using an NanoSight NS300 instrument (Malvern Instruments, Amesbury, UK) according to the manufacturer's instructions. The size distribution and concentration of the secreted vesicles were recorded. Meantime, cells left on the culture plate were trypsinized and counted by Countless II (Life Science). The exosomes released/cell were then determined. 


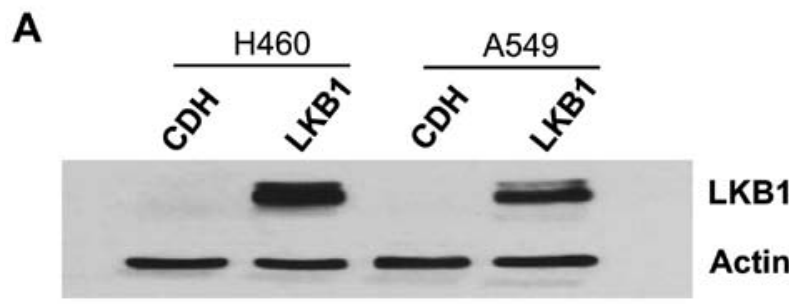

B
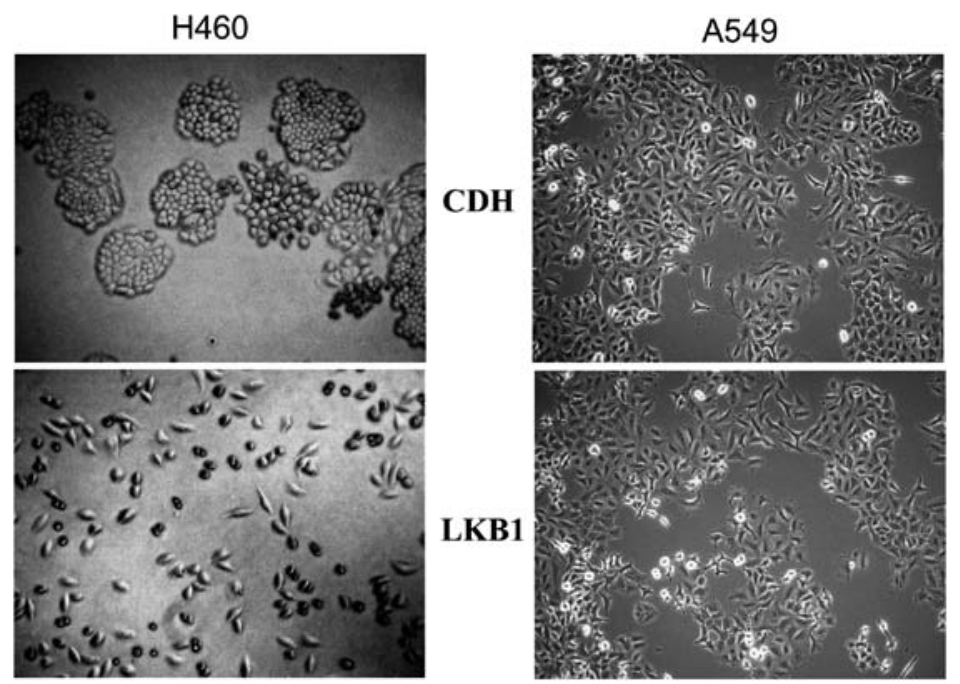

Figure 1. Restoration of LKB1 markedly alters morphology and clustering of H460 lung cancer cells. (A) LKB1 expression was analyzed by western blotting in H460 and A549 cells with stable expression of either the pCDH lentiviral vector (CDH) or LKB1. Actin served as a loading control. (B) Phase-contrast images of $\mathrm{H} 460$ and A549 cells growing in a plastic 6-well plate. CDH: cells with stable expression of pCDH lentiviral vector. LKB1, cells with stable expression of LKB1. Magnification, $\mathrm{x} 40$.

Exosome uptake test. Exosomes were labeled with the red fluorescent dye PKH26 (Sigma-Aldrich) according to the manufacturer's recommendation. Briefly, isolated exosomes were resuspended in $2 \mathrm{ml}$ Diluent $\mathrm{C}$ solution containing $1 \mu \mathrm{l}$ PKH26. After $5 \mathrm{~min}$ of incubation, $2 \mathrm{ml}$ of $1 \%$ bovine serum albumin was added to stop the labeling reaction. The exosome suspension was centrifuged at $169,000 \mathrm{x} \mathrm{g}$ for $90 \mathrm{~min}$ to pellet the labeled exosomes, followed by resuspension in $1 \mathrm{ml}$ of complete medium. Resuspended exosomes were counted, and then added to $\mathrm{H} 460$ cells at a dose of 100 exosomes/receipt cell for $18 \mathrm{~h}$ of incubation. 4',6-Diamidino-2-phenylindone (DAPI; Invitrogen) was used for nuclear staining. Cell images were captured using an inverted microscope (EVOS; NY).

RNA extraction and RT-qPCR. Total RNA in cells or isolated exosomes was extracted using mirVana miRNA Isolation kit (Ambion, Austin, TX, USA) and reverse transcribed to cDNA using TaqMan Advanced MicroRNA cDNA Synthesis kit (Thermo Fisher Scientific Waltham, MA, USA). qPCR was carried out using TaqMan Advanced MicroRNA Assay kit (Thermo Fisher Scientific) on the Applied Biosystems 7500 (Applied Biosystems, Foster City, CA, USA) following the manufacturer's instructions. The relative levels of let $7 \mathrm{~b}, \mathrm{miR}-$ $125 \mathrm{a}$ or miR-126 were normalized to that of hsa-miR-191-5p (for cellular samples) or that of U6 (for exosome samples).

Statistical analysis. Results are expressed as mean \pm SEM. Statistical significance was determined by a two-sided Student's t-test. $\mathrm{P}<0.05$ was considered statistically significant.

\section{Results}

Restoration of LKB1 in LKB1-deficient lung cancer cells markedly promotes cell motility. In an attempt to investigate the role of LKB1 in exosome secretion and the related cellular signaling, we stably restored LKB1 expression in LKB1-dificient H460 lung cancer cells (10) by lentiviral transduction (Fig. 1A). We noted that restoration of LKB1 led to a marked change in cell morphology in the plastic plate (left panels, Fig. 1B). While H460 cells with stable expression of the lentiviral vector $\left(\mathrm{H} 460^{\mathrm{CDH}}\right)$ were similar to the parental $\mathrm{H} 460$ cells that exhibited a round shape and were clustered, $\mathrm{H} 460$ cells with stable expression of LKB1 $\left(\mathrm{H}_{4} 60^{\mathrm{LKB} 1}\right)$ had an elongated shape and were well-dispersed (Fig. 1B), implying the increased motility of the $\mathrm{H} 460^{\mathrm{LKB} 1}$ cells. Indeed, compared with the $\mathrm{H} 460^{\mathrm{CDH}}$ cells, $\mathrm{H} 460^{\mathrm{LKB} 1}$ cells exhibited greatly increased migration ability (Fig. 2A). We then decided to confirm this finding using another LKB1-deficient lung cancer cell line: A549. While restoration of LKB1 in A549 cells did not cause an obvious change in cell morphology (right panels, Fig. 1B), it did greatly increase A549 cell migration (Fig. 2B). As LKB1 is known to suppress tumor cell growth, we examined the effects of LKB1 restoration on lung cancer cell growth by performing soft-agar colony formation assay. As expected, LKB1 restoration greatly suppressed H460 colony formation (Fig. 3A). A549 cells did not show measurable colony formation in our experiments. We therefore conducted MTS cell proliferation assay for this cell line. LKB1 restoration significantly decreased A549 cell proliferation (Fig. 3B). In accordance with its effect on colony 
A
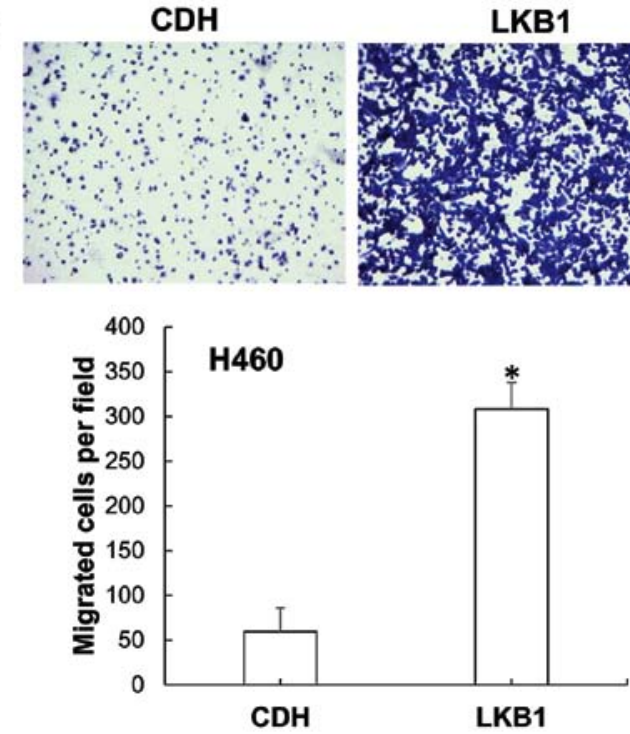

B

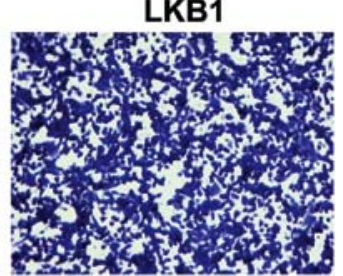

LKB1
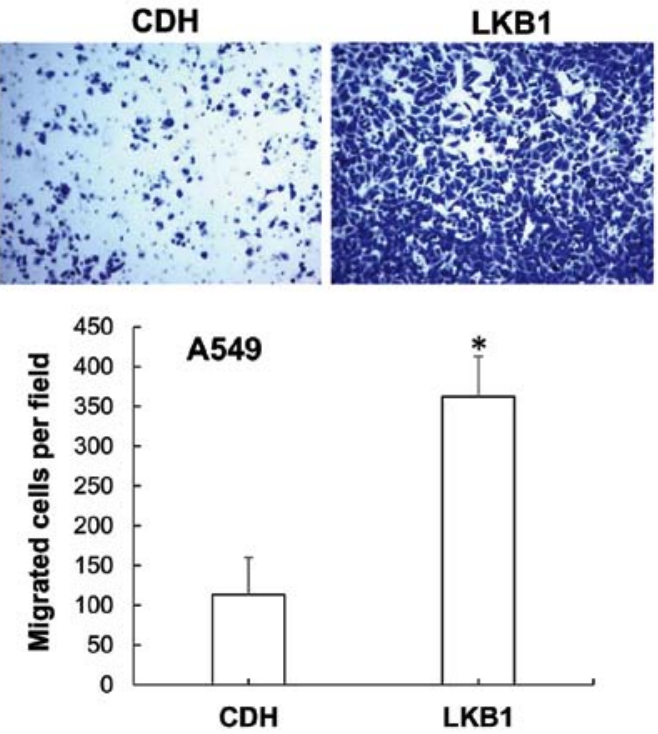

Figure 2. LKB1 restoration greatly increases the migration of both $\mathrm{H} 460$ and A549 cells. Shown on the top are the representative images of migrated (A) H460 or (B) A549 cells with stable expression of either the $\mathrm{pCDH}$ lentiviral vector $(\mathrm{CDH})$ or LKB1. Quantitative migration ability is presented as the number of migrated cells/field. Values are mean \pm SEM of 3 separate experiments; ${ }^{*} \mathrm{P}<0.001$ (Student's t-test).

A

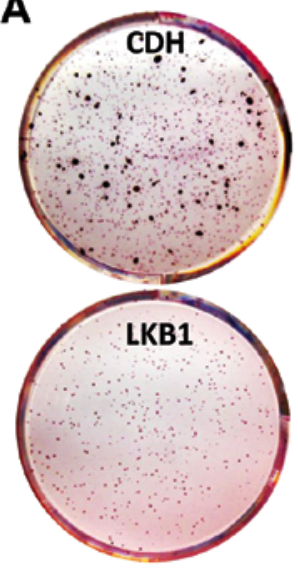

B

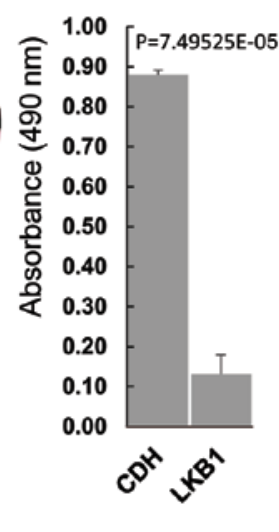

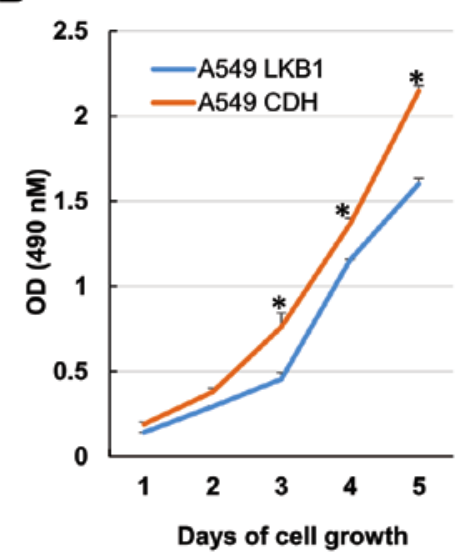

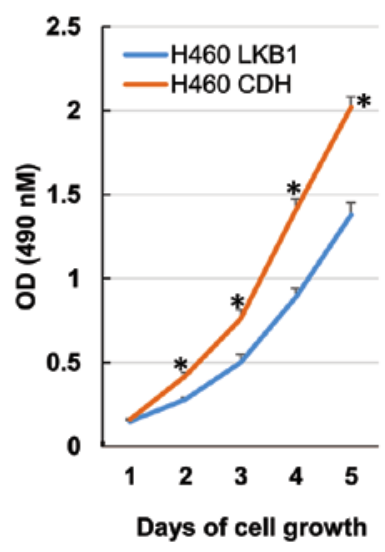

Figure 3. LKB1 restoration inhibits the growth of both $\mathrm{H} 460$ and A549 lung cancer cells. (A) Colony formation assay of H460 ${ }^{\mathrm{CDH}}$ and $\mathrm{H} 460^{\mathrm{LKB} 1}$ stable cell pools. Representative images of colonies formed in the soft-agar plate are shown on the left and quantification of colony formation is shown on the right. Values are mean \pm SEM of 3 separate experiments. (B) MTS cell proliferation assay of A549 ${ }^{\mathrm{CDH}}, \mathrm{A} 549^{\mathrm{LKB} 1}, \mathrm{H} 460^{\mathrm{CDH}}$ and $\mathrm{H} 460^{\mathrm{LKB} 1}$ stable cell pools. Values are mean \pm SEM of 4 separate experiments; ${ }^{*} \mathrm{P}<0.01$ (Student's t-test).

formation, LKB1 restoration also significantly decreased H460 cell proliferation (Fig. 3B).

LKB1 greatly enhances the release of exosomes by H460 lung cancer cells. We then analyzed the effect of LKB1 expression on the secretion of exosomes by $\mathrm{H} 460$ cells, which was the original aim of the present study. We isolated extracellular vesicles from conditioned media and analyzed them using NanoSight NS300 instrument. As shown in Fig. 4, LKB1 greatly increased the release of exosomes by $\mathrm{H} 460$ cells.

Exosomes secreted by H460-LKB1 cells have enhanced ability to promote cell migration. Exosomes have been shown to be involved in cell migration (11), which can be either promoting or inhibiting, depending on the contents contained in the extracellular vesicles. We therefore tested the difference in exosomes from either $\mathrm{H} 460^{\mathrm{CDH}}$ or $\mathrm{H} 460^{\mathrm{LKB} 1}$ cells in impacting motility of receipt cells. As exosomes can target both tumor cells and tumor-associated cells in both distant sites and local areas (7), we simply used $\mathrm{H} 460$ parental cells for testing the differential effects of these exosomes on cell motility of receipt cells. As shown in Fig. 5, exosomes isolated from the conditioned media of either $\mathrm{H} 460^{\mathrm{CDH}}$ or $\mathrm{H} 460^{\mathrm{LKB} 1}$ were readily taken up by $\mathrm{H} 460$ cells. Notably, in comparison with those that received $\mathrm{H} 460^{\mathrm{CDH}}$ exosomes, cells that received $\mathrm{H} 460^{\mathrm{LKB} 1}$ exosomes had much higher motility (Fig. 6). These results suggest that restoration of LKB1 in H460 cells not only stimulated the secretion of exosomes, but also changed the feature of exosomes towards an increase in migration-promoting ability.

LKBI decreases the levels of migration-suppressing miRNAs both in cells and in exosomes. We then investigated how restoration of LKB1 promotes $\mathrm{H} 460$ lung cell migration and why exosomes from $\mathrm{H} 460^{\mathrm{LKB} 1}$ cells have higher activity in 
A

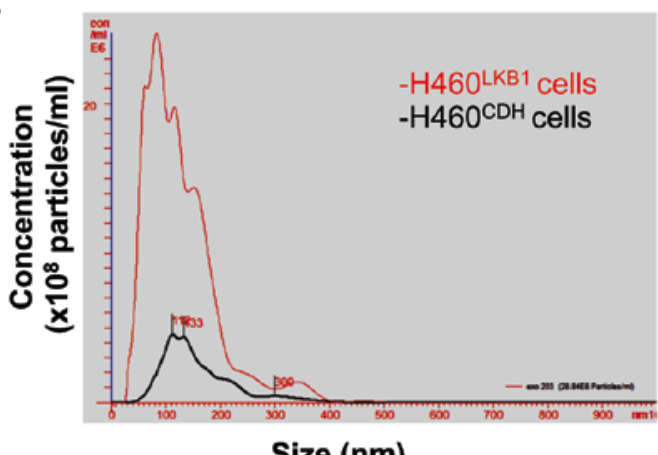

Size $(\mathrm{nm})$

B

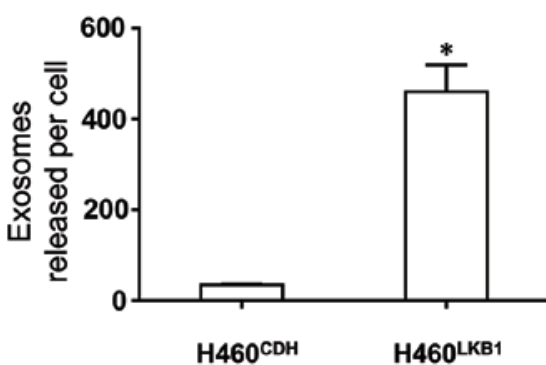

Figure 4. LKB1 restoration greatly increases the release of exosomes. The number of exosomes released from $\mathrm{H} 460^{\mathrm{CDH}}$ and $\mathrm{H} 460^{\mathrm{LKB} 1}$ cells was determined by NanoSight tracking analysis (NTA). (A) Representative NTA plots showing the size distribution and total number of exosomes released from $\mathrm{H} 460^{\mathrm{CDH}}$ and $\mathrm{H} 460^{\mathrm{LKB} 1}$ cells. (B) The number of exosomes released per $\mathrm{H} 460^{\mathrm{CDH}}$ or $\mathrm{H} 460^{\mathrm{LKB1}}$ cell (the total number of exosomes/the total number of cells). Data represents mean $\pm \mathrm{SEM} ;{ }^{*} \mathrm{P}<0.005$ vs. $\mathrm{H} 460^{\mathrm{CDH}}$ cells.

promoting migration of receipt cells. As miRNAs are the essential components in exosomes, we examined the effect of LKB1 restoration on the levels of miRNAs both in cells and in exosomes. We focused on let7b, miR-125a and miR-126, all of which are known to regulate cell migration and be secreted in
A Exosomes ${ }^{\mathrm{CDH}}$

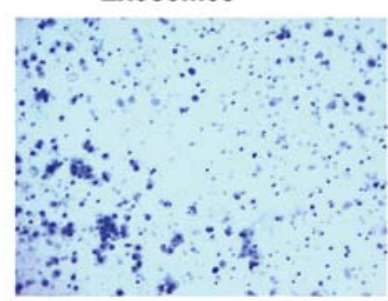

B

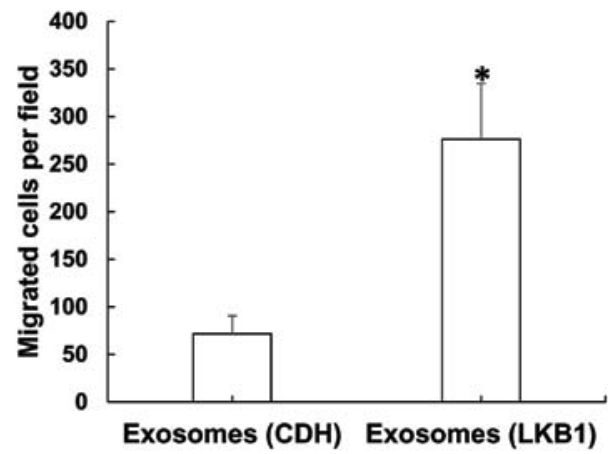

Exosomes LKB

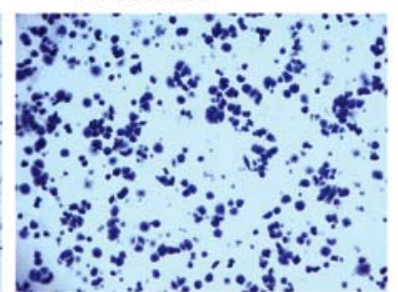

Exosomes (LKB1)
Figure 6. Exosomes secreted by $\mathrm{H} 460^{\mathrm{LKB} 1}$ cells have higher ability of promoting cell migration. H460 parental cells were treated with isolated exosomes (at a dose of 100 exosomes/cell) from the conditioned media of either $\mathrm{H} 460^{\mathrm{CDH}}$ cells (Exosomes ${ }^{\mathrm{CDH}}$ ) or $\mathrm{H} 460^{\mathrm{LKB} 1}$ cells (Exosomes ${ }^{\mathrm{LKB1}}$ ) for 1 day, followed by Transwell cell migration assay. Representative images of cell migration are shown (A) and quantitative migration ability is presented as the number of migrated cells/field (B). Values are mean \pm SEM of 3 separate experiments; ${ }^{*} \mathrm{P}<0.001$ (Student's t-test).

exosomes (12-16). As shown in Fig. 7, LKB1 restoration caused a significant decrease in the cellular levels of both miR-125a and miR-126, but had no significant effect on the cellular level of let7b. In exosomes, the levels of let7b and miR-126 were significantly reduced by LKB1 restoration, whereas the level of miR-125a was not significantly changed. These results suggest that LKB1 promotes cell migration, at least partly

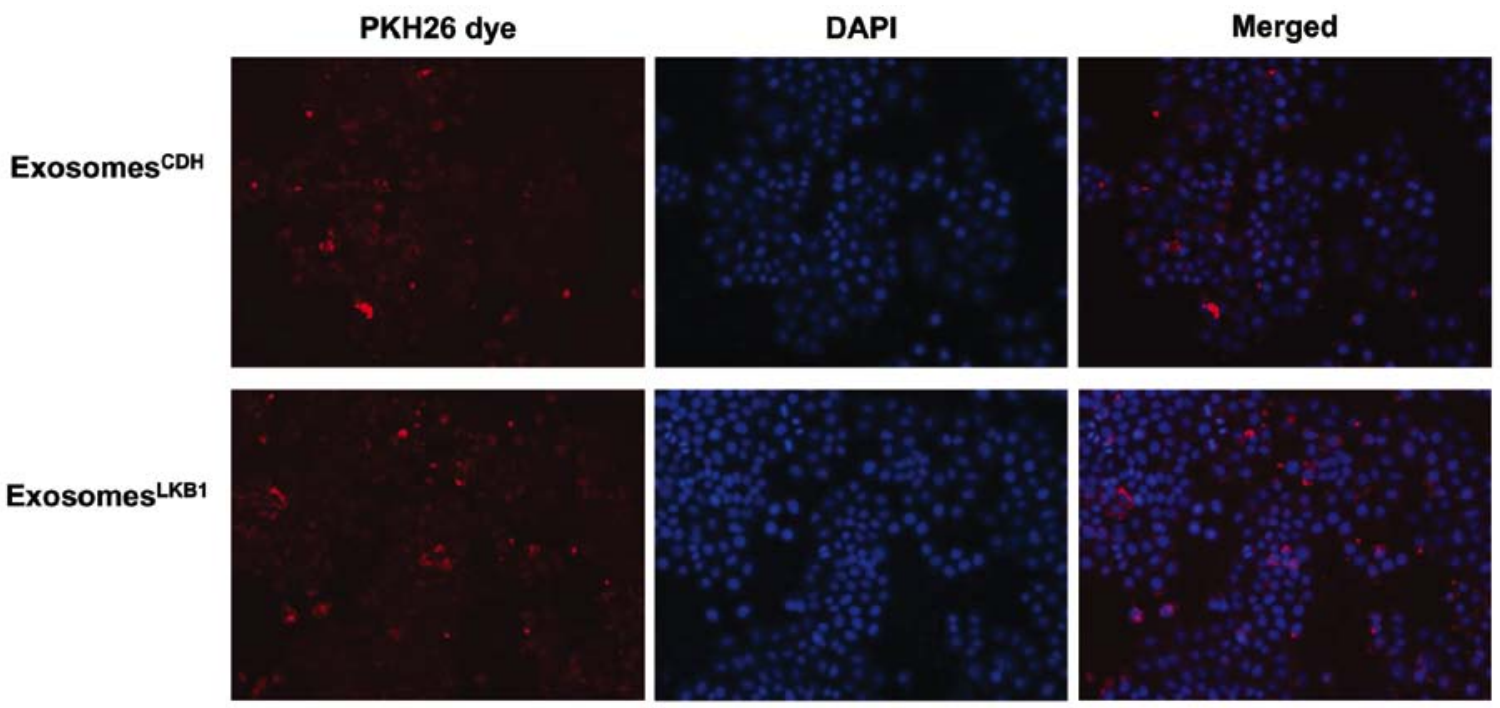

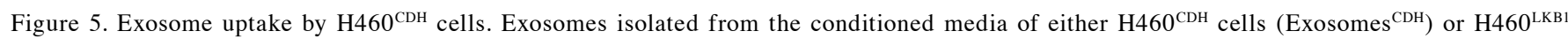
cells (Exosomes ${ }^{\mathrm{LKB}}$ ) were labeled with $\mathrm{PKH} 26$ red fluorescent dye. $\mathrm{H} 460$ cells with stable expression of the pCDH lentiviral vector (H460 ${ }^{\mathrm{CDH}}$ ) were treated with PKH26-labeled exosomes and exosome uptake was analyzed by imaging using fluorescence microscopy. DAPI staining shows cell nuclei. Magnification, x100. 
A

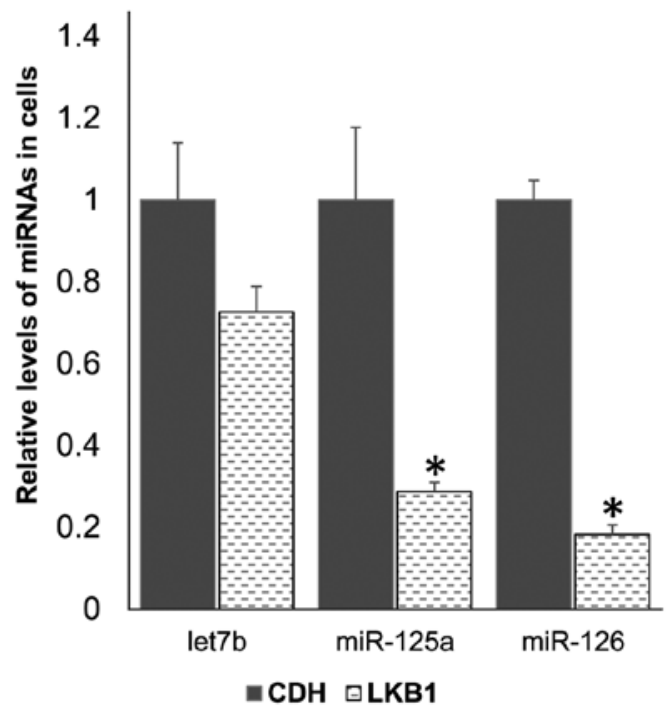

B

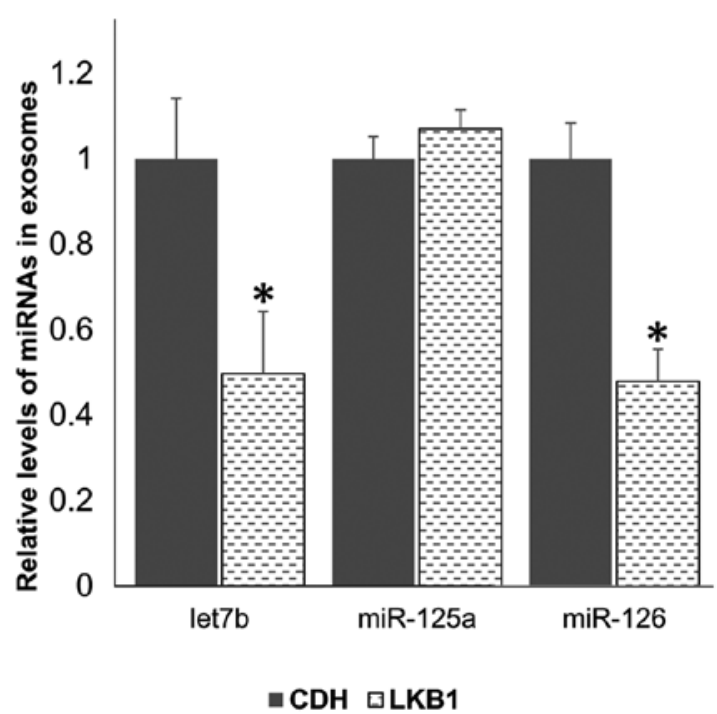

Figure 7. Levels of let7b, miR-125a and miR-126 are decreased both in (A) cells and in (B) exosomes upon LKB1 restoration in H460 cells. The levels of miRNAs in $\mathrm{H} 460^{\mathrm{CDH}}$ and $\mathrm{H} 460^{\mathrm{LKB} 1}$ cells were analyzed by RT-qPCR. Data are presented in relative to the level of each miRNA (set as 1 ) from H460 ${ }^{\mathrm{CDH}}$ cells. Values are mean \pm SEM of 3 separate experiments; * $\mathrm{P}<0.01$ (Student's t-test).

through downregulating the levels of migration-suppressing miRNAs both in cells and in exosomes.

\section{Discussion}

LKB1 is a critical factor in controlling cell polarity and morphology $(17,18)$, both of which are implicated in cell migration. Indeed, LKB1 promotes the migration of neuron $(19,20)$ and endothelial cells (21). The role of LKB1 in cancer cell migration, however, has been elusive. While some studies have shown that LKB1 inhibits cancer cell migration and tumor metastasis (22-25), others have reported that LKB1 positively regulates the signaling of PAKs at the cell membrane region and stimulates the formation of protrusive membrane structures (lamellipodia) (26), suggesting a motility-stimulating role for LKB1. Indeed, two recent studies showed that LKB1 re-expression enhanced directional migration of the H157 lung cancer cell line and HeLa cervical cancer cell line, both of which lack LKB1 expression $(27,28)$. The present study corroborated the stimulating effect of LKB1 restoration in motility of LKB1-deficient lung cancer cells. In addition, the present study revealed a new mechanism by which LKB1 promotes cancer cell migration, i.e., downregulating the levels of migration-suppressing microRNAs (miRNAs) in both cells and exosomes. Notably, although LKB1 restoration enhanced motility of both H460 and A549 cells, it changed H460 cells from a round epithelial shape to an elongated mesenchymallike shape, but had little effect on A549 cell morphology. These results suggest that LKB1 promotes the motility of H460 and A549 cells probably by different mechanisms. LKB1 may stimulate epithelial-mesenchymal transition in $\mathrm{H} 460$ cells on the basis of the morphological change aforementioned, whereas in A549 cells, it may increase directional migration, a similar effect for LKB1 restoration reported in H157 cells (27). While the underlying molecular mechanisms remain to be elucidated, it is interesting to note that $\mathrm{H} 460$ is a cell line derived from large cell lung carcinoma, whereas both
A549 and H157 are non-small cell lung adenocarcinoma cell lines. Taken together, the exact role of LKB1 in cell migration is likely dependent upon cell type and the physiological and pathological conditions.

While LKB1 is well-known for regulating multiple critical signaling pathways, such as AMPK/mTOR and p53 pathways, little is known concerning the involvement of LKB1 in exosome-mediated cellular signaling. The present study demonstrates that LKB1 greatly stimulates the release of exosomes by H460 cells. Our finding concerning LKB1 in stimulating exosome release is new, but may not be surprising, given that LKB1 signaling is shown to be involved in protein trafficking and secretion $(2,29)$. Notably, LKB1 was shown to interact with Rab7 GTPase and regulates RAB7-mediated protein trafficking (30). Rab7 is involved in exosome secretion of endothelial (31) and melanoma cells (32). In addition, LKB1 is known to be implicated in insulin secretion although the underlying mechanisms remain to be elucidated (33). Moreover, LKB1 was reported to regulate microtubule-dependent trafficking of ABCB11 in hepatocytes (34). Intriguingly, microtubule affinity-regulating kinases (MARKs) are downstream targets of LKB1 and play essential roles in microtubule skeleton organization $(35,36)$. As such, it may be important to investigate in future research whether Rab7 and/or MARK proteins are involved in LKB1-stimulated exosome release in H460 lung cancer cells.

Another interesting finding from the present study is that exosomes from $\mathrm{H} 460$ cells with LKB1 restoration, in comparison with those released by LKB1-deficient H460 cells, have highly increased ability in promoting migration of receipt cells. This is likely due to the decreased levels of tumor-suppressing miRNAs (e.g., let7b and miRNA-126) in exosomes from H460 cells upon LKB1 restoration. Taken together, the present study revealed a new role for LKB1 in promoting cell motility, at least partly by downregulating migration-suppressing miRNA expression and exosome secretion. One of the proposed therapeutic strategies for treating lung cancer with loss of LKB1 function is to restore 
LKB1 expression and/or signaling. However, our findings suggest that this strategy may be a double-edged sword with a suppressing role in tumor cell growth and possibly a promoting role in cancer cell invasiveness.

\section{Acknowledgements}

The present study was supported by a start-up fund of Wright State University and by NCI 1R01CA193264-01 to W.W.L.

\section{References}

1. Korsse SE, Peppelenbosch MP and van Veelen W: Targeting LKB1 signaling in cancer. Biochim Biophys Acta 1835: 194-210, 2013.

2. Gao Y, Ge G and Ji H: LKB1 in lung cancerigenesis: A serine/threonine kinase as tumor suppressor. Protein Cell 2: 99-107, 2011

3. Ylikorkala A, Rossi DJ, Korsisaari N, Luukko K, Alitalo K, Henkemeyer M and Mäkelä TP: Vascular abnormalities and deregulation of $V E G F$ in $L k b 1$-deficient mice. Science 293 1323-1326, 2001

4. Hemminki A, Markie D, Tomlinson I, Avizienyte E, Roth S, Loukola A, Bignell G, Warren W, Aminoff M, Höglund P, et al: A serine/threonine kinase gene defective in Peutz-Jeghers syndrome. Nature 391: 184-187, 1998.

5. Marcus AI and Zhou W: LKB1 regulated pathways in lung cancer invasion and metastasis. J Thorac Oncol 5: 1883-1886, 2010.

6. Gangoda L, Boukouris S, Liem M, Kalra H and Mathivanan S: Extracellular vesicles including exosomes are mediators of signal transduction: Are they protective or pathogenic? Proteomics 15 260-271, 2015

7. Vader P, Breakefield XO and Wood MJ: Extracellular vesicles: Emerging targets for cancer therapy. Trends Mol Med 20: 385-393, 2014.

8. Zhao L, Liu W, Xiao J and Cao B: The role of exosomes and 'exosomal shuttle microRNA' in tumorigenesis and drug resistance. Cancer Lett 356: 339-346, 2015.

9. Elkhadragy L, Chen M, Miller K, Yang MH and Long W: A regulatory BMI1/let-7i/ERK3 pathway controls the motility of head and neck cancer cells. Mol Oncol 11: 194-207, 2017.

10. Blanco R, Iwakawa R, Tang M, Kohno T, Angulo B, Pio R, Montuenga LM, Minna JD, Yokota J and Sanchez-Cespedes M: A gene-alteration profile of human lung cancer cell lines. Hum Mutat 30: 1199-1206, 2009.

11. Weidle UH, Birzele F, Kollmorgen G and Rüger R: The multiple roles of exosomes in metastasis. Cancer Genomics Proteomics 14 $1-15,2017$.

12. $\mathrm{Hu} \mathrm{G}$, Drescher KM and Chen XM: Exosomal miRNAs: Biological properties and therapeutic potential. Front Genet 3: $56,2012$.

13. Spolverini A, Fuchs G, Bublik DR and Oren M: let-7b and let-7c microRNAs promote histone $\mathrm{H} 2 \mathrm{~B}$ ubiquitylation and inhibit cell migration by targeting multiple components of the H2B deubiquitylation machinery. Oncogene: Jun 12, 2017 (Epub ahead of print). doi: 10.1038/onc.2017.187.

14. Jiang L, Huang Q, Zhang S, Zhang Q, Chang J, Qiu X and Wang E: Hsa-miR-125a-3p and hsa-miR-125a-5p are downregulated in non-small cell lung cancer and have inverse effects on invasion and migration of lung cancer cells. BMC Cancer 10: 318, 2010.

15. Wang J, Yan F, Zhao Q, Zhan F, Wang R, Wang L, Zhang Y and Huang $\mathrm{X}$ : Circulating exosomal miR-125a-3p as a novel biomarker for early-stage colon cancer. Sci Rep 7: 4150, 2017.

16. Ebrahimi F, Gopalan V, Smith RA and Lam AK: miR-126 in human cancers: Clinical roles and current perspectives. Exp Mol Pathol 96: 98-107, 2014.

17. Martin SG and St Johnston D: A role for Drosophila LKB1 in anterior-posterior axis formation and epithelial polarity. Nature 421: 379-384, 2003.
18. Baas AF, Kuipers J, van der Wel NN, Batlle E, Koerten HK, Peters PJ and Clevers HC: Complete polarization of single intestinal epithelial cells upon activation of LKB1 by STRAD. Cell 116: 457-466, 2004.

19. Asada N, Sanada K and Fukada Y: LKB1 regulates neuronal migration and neuronal differentiation in the developing neocortex through centrosomal positioning. J Neurosci 27: $11769-11775,2007$

20. Asada N and Sanada K: LKB1-mediated spatial control of GSK3beta and adenomatous polyposis coli contributes to centrosomal forward movement and neuronal migration in the developing neocortex. J Neurosci 30: 8852-8865, 2010.

21. Ohashi K, Ouchi N, Higuchi A, Shaw RJ and Walsh K: LKB1 deficiency in Tie2-Cre-expressing cells impairs ischemiainduced angiogenesis. J Biol Chem 285: 22291-22298, 2010.

22. Li J, Liu J, Li P, Mao X, Li W, Yang J and Liu P: Loss of LKB1 disrupts breast epithelial cell polarity and promotes breast cancer metastasis and invasion. J Exp Clin Cancer Res 33: 70, 2014.

23. Chan KT, Asokan SB, King SJ, Bo T, Dubose ES, Liu W, Berginski ME, Simon JM, Davis IJ, Gomez SM, et al: LKB1 loss in melanoma disrupts directional migration toward extracellular matrix cues. J Cell Biol 207: 299-315, 2014.

24. Ji H, Ramsey MR, Hayes DN, Fan C, McNamara K, Kozlowski P, Torrice C, Wu MC, Shimamura T, Perera SA, et al: LKB1 modulates lung cancer differentiation and metastasis. Nature 448: 807-810, 2007.

25. Roy BC, Kohno T, Iwakawa R, Moriguchi T, Kiyono T, Morishita K, Sanchez-Cespedes M, Akiyama T and Yokota J: Involvement of LKB1 in epithelial-mesenchymal transition (EMT) of human lung cancer cells. Lung Cancer 70: 136-145, 2010.

26. Zhang S, Schafer-Hales K, Khuri FR, Zhou W, Vertino PM and Marcus AI: The tumor suppressor LKB1 regulates lung cancer cell polarity by mediating cdc 42 recruitment and activity. Cancer Res 68: 740-748, 2008.

27. Konen J, Wilkinson S, Lee B, Fu H, Zhou W, Jiang Y and Marcus AI: LKB1 kinase-dependent and -independent defects disrupt polarity and adhesion signaling to drive collagen remodeling during invasion. Mol Biol Cell 27: 1069-1084, 2016.

28. Wilkinson S, Hou Y, Zoine JT, Saltz J, Zhang C, Chen Z, Cooper LA and Marcus AI: Coordinated cell motility is regulated by a combination of LKB1 farnesylation and kinase activity. Sci Rep 7: 40929, 2017.

29. Gan RY and Li HB: Recent progress on liver kinase B1 (LKB1): Expression, regulation, downstream signaling and cancer suppressive function. Int J Mol Sci 15: 16698-16718, 2014.

30. Okon IS, Coughlan KA, Zhang C, Moriasi C, Ding Y, Song P, Zhang W, Li G and Zou MH: Protein kinase LKB1 promotes RAB7-mediated neuropilin-1 degradation to inhibit angiogenesis. J Clin Invest 124: 4590-4602, 2014.

31. Jaé N, McEwan DG, Manavski Y, Boon RA and Dimmeler S: Rab7a and Rab27b control secretion of endothelial microRNA through extracellular vesicles. FEBS Lett 589: 3182-3188, 2015.

32. Peinado H, Alečković M, Lavotshkin S, Matei I, Costa-Silva B, Moreno-Bueno G, Hergueta-Redondo M, Williams C, GarcíaSantos G, Ghajar C, et al: Melanoma exosomes educate bone marrow progenitor cells toward a pro-metastatic phenotype through MET. Nat Med 18: 883-891, 2012.

33. Granot Z, Swisa A, Magenheim J, Stolovich-Rain M, Fujimoto W, Manduchi E, Miki T, Lennerz JK, Stoeckert CJ Jr, Meyuhas O, et al: LKB1 regulates pancreatic beta cell size, polarity, and function. Cell Metab 10: 296-308, 2009.

34. Homolya L, Fu D, Sengupta P, Jarnik M, Gillet JP, Vitale-Cross L Gutkind JS, Lippincott-Schwartz J and Arias IM: LKB1/AMPK and PKA control ABCB11 trafficking and polarization in hepatocytes. PLoS One 9: e91921, 2014.

35. Drewes G, Ebneth A and Mandelkow EM: MAPs, MARKs and microtubule dynamics. Trends Biochem Sci 23: 307-311, 1998.

36. Kojima Y, Miyoshi H, Clevers HC, Oshima M, Aoki M and Taketo MM: Suppression of tubulin polymerization by the LKB1-microtubule-associated protein/microtubule affinityregulating kinase signaling. J Biol Chem 282: 23532-23540, 2007. 